\title{
Molecular Investigation of Brain Tumors Progressing During Pregnancy or Postpartum Period: The Association Between Tumor Type, Their Receptors, and the Timing of Presentation
}

\section{Saeko Ichimura}

Keio University School of Medicine

\section{Kentaro Ohara}

Keio University School of Medicine

Maya Kono

Tokyo Metropolitan Children's Medical Center

Katsuhiro Mizutani

Japanese Red Cross Ashikaga Hospital

\section{Yohei Kitamura}

Tokyo Saiseikai Central Hospital

Isako Saga

Saitama City Hospital

Ryuichi Kanai

Eiju General Hospital

\section{Takenori Akiyama}

Keio University School of Medicine

Masahiro Toda

Keio University School of Medicine

Michihiro Kohno

Tokyo Metropolitan Police Hospital

Kazunari Yoshida

Keio University School of Medicine

Hikaru Sasaki ( $\square$ hsasaki@keio.jp )

Keio University School of Medicine https://orcid.org/0000-0001-7380-1931

\section{Research Article}

Keywords: Brain tumor, pregnancy, postpartum period, molecular, investigation

Posted Date: February 12th, 2021 
DOl: https://doi.org/10.21203/rs.3.rs-190193/v1

License: (c) (1) This work is licensed under a Creative Commons Attribution 4.0 International License. Read Full License 


\section{Abstract}

Brain tumors are frequently present during pregnancy; however, the mechanism has not been well elucidated. Purpose of this study is to investigate the influence of molecular genetic factors on the progression of brain tumors during pregnancy or the postpartum period.

Twelve cases of brain tumors that presented during pregnancy or postpartum period were included: five gliomas, three meningiomas, two vestibular schwannomas, and two chordomas. Tumor samples were investigated by metaphase comparative genomic hybridization and immunohistochemistry, for chromosomal copy number aberration (CNA) and receptor expression of sex hormones and growth factors.

The results were correlated with the timing of tumor presentation in relation to the stage of pregnancy. EGFR, VEGFR-1/2, AR, and c-Myc were expressed in gliomas, PgR, ER, HER-2, VEGFR-1, EGF and VEGFR2 in meningiomas, VEGFR-1 in vestibular schwannomas, and EGFR, VEGFR-1/2, and c-Myc in chordomas. The CNAs of the tumors varied. Four of the five gliomas presented in the 2nd trimester, all three meningiomas in the 3rd trimester or postpartum period, and both of the two schwannomas in the late 2 nd trimester. Expression of VEGFR-1/2 and EGFR was observed regardless of the timing of tumor presentation, whereas female hormone receptors and HER-2 were exclusively found in meningiomas. Interestingly, one anaplastic astrocytoma (IDH mut, non-codeleted) that progressed from precedent grade 2 tumor harbored amplification of the MYClocus.

Progression of brain tumors during pregnancy is associated with various growth factors as well as sex hormones. The timing of presentation is likely dependent on molecular receptors specific to each tumor type.

\section{Introduction}

Primary brain tumors in women of child-bearing age are rare, but it has been recognized that brain tumors often manifest or progress during pregnancy.

In 1938, Cushing and Eisenhardt reported cases of meningiomas, for the first time, which had symptom onset during pregnancy, remission during the postpartum period, and relapse with subsequent pregnancies [4]. Enlargement of meningioma may be related to pregnancy or menstrual cycles due to sex steroid hormones [20]. In fact, progesterone and estrogen independently stimulate meningioma growth in vitro [20], and in most studies, meningiomas histologically show immunoreactivity to both PgR and ER, although the positivity rate for PgR is usually higher than that for ER [20]. Similar observations have been made for other primary brain tumors. A review paper reported 223 cases of primary brain tumors with first manifestation or acceleration of symptoms during pregnancy, including gliomas, meningiomas, vestibular schwannomas, and hemangioblastomas/hemangiomas in order of frequency [19]. Indeed, a previous study demonstrated that pregnancy increases the radiological growth rates of grade 2 gliomas [16]. 
In a population-based study in Norway, breast cancer that presented during pregnancy or lactation was associated with larger tumor size and higher clinical staging than those presented before pregnancy [18]. Importantly, the majority of pregnancy-associated breast tumors were negative for hormone receptors; however, HER-2 was expressed in nearly half of the cases.

Thus, pregnancy can affect the growth of various cancers, including brain tumors. Although the association between sex hormones and other growth factors has been implicated, the mechanism of growth during pregnancy has not been well elucidated. For example, maternal intravascular volume may also be involved as it increases with advancing gestational age [2].

The purpose of this study was to investigate the influence of molecular genetic factors on the progression of brain tumors during pregnancy or the postpartum period.

\section{Methods}

\section{Tissue samples}

Specimens of 12 brain tumors that manifested or progressed during pregnancy or postpartum period were collected from Keio University Hospital and collaborative hospitals from 2010 to 2017: five were gliomas, three were meningiomas, two were chordomas, and two were vestibular schwannomas based on the local pathological diagnosis according to the World Health Organization (WHO) criteria [12].

This translational research was approved by the Institutional Review Board at each hospital, and experiments were performed at Keio University School of Medicine.

Written informed consent was obtained from all 12 patients.

\section{Clinical data}

Clinical data were obtained from the patients' records, including age at diagnosis, symptom, timing of tumor manifestation, treatment, and institutional histopathological diagnosis [12, 13]. The timing of tumor manifestation was analyzed in relation to the stage of pregnancy; the first trimester was defined as the period between week 1 and week 12, the second trimester between week 13 to week 28 , the third trimester between week 29 and week 40, and the postpartum period defined within six weeks after delivery. Pathological diagnoses of tumors was reviewed by KO according to the WHO criteria in 2016 [13].

\section{Immunohistochemistry}

Expression of sex hormone receptors, including estrogen receptor (ER), progesterone receptor (PgR), and androgen receptor (AR), as well as that of growth factor receptors, including epidermal growth factor receptor (EGFR), human epidermal growth factor receptor 2 (HER-2), and vascular endothelial growth factor receptor (VEGFR) -1/2 was assessed by immunohistochemistry. Expression of c-Myc was also assessed by immunohistochemistry. 
Three um-thick FFPE sections were stained with anti-human ER antibody (1D5, 1:100, Envision FLEX-ER ${ }^{\mathrm{TM}}$, Dako, Glostrup, Denmark) for ER, anti-human PgR antibody (PgR636, 1:500, Envision FLEX-PgR ${ }^{\text {TM }}$, Dako) for PgR, anti-human AR antibody (AR27, 1:50, Novocastra, Newcastle upon Tyne, UK) for AR, anti-human EGFR antibody (31G7, 1:50, Nichirei,Tokyo, Japan) for EGFR, anti-human HER-2 antibody (CB11, 1:100, Novocastra) for HER-2, anti-human VEGFR-1/Flt-1 antibody (AF321, 1:100, R\&D Systems, Minneapolis, MN, USA) for VEGFR-1, anti-human VEGFR-2 antibody (55B11, 1:200, Cell Signaling Technology, Danvers, MA, USA) for VEGFR-2, and with anti-human c-Myc antibody (MC045, 1:500, Abcam, Cambridge, UK) for c-Myc. For the evaluation of ER, FFPE sections from mammary glands resected in 2006 were used as positive controls; staining of $>5 \%$ nuclei was deemed a positive. For the evaluation of PgR, FFPE sections from meningiomas resected in 2010 were used as positive controls; staining of $>5 \%$ nuclei was deemed a positive. For the evaluation of AR, FFPE sections from brain metastasis of prostate cancer resected in 2011 were used as positive controls; staining of $>5 \%$ nuclei was deemed a positive. For the evaluation of EGFR, FFPE sections from glioblastoma resected in 2011 were used as positive controls; staining of cell membrane in $>5 \%$ of cells was deemed a positive. For the evaluation of HER-2, FFPE sections from brain metastasis of breast cancer resected in 2010 were used as positive controls; staining of cell membrane in $>5 \%$ of cells was deemed a positive. For the evaluation of VEGFR-1/2, FFPE sections from glioblastoma resected in 2011 were used as positive controls; staining of vascular endothelium was determined a positive $(1+)$ and staining of both vascular endothelium and cytoplasm as positive (2+). For the evaluation of c-Myc, FFPE sections from anaplastic oligodendroglioma resected in 2012 were used as positive controls and staining of $>10 \%$ nuclei and cytoplasm was deemed a positive.

\section{Genetic analyses}

Tumor DNA was extracted from microdissected pieces of formalin-fixed paraffin-embedded (FFPE) tissue [21]. For tissue microdissection, intermixed non-neoplastic glial/vascular cells and hemorrhagic/necrotic regions were excluded. This was based on hematoxylin and eosin staining and MIB-1

immunohistochemistry (Dako) on consecutive sections [10].

Chromosomal number aberrations (CNAs) were assessed by metaphase comparative genomic hybridization $(\mathrm{CGH})$ as described previously $[8,14]$. In brief, crude tumor DNA from FFPE tissue was amplified by degenerate oligonucleotide primed-polymerase chain reaction (DOP-PCR) and labeled with another DOP-PCR using digoxigenin (DIG)-11-dUTP (Roche, Mannheim, Germany). The reference DNA was amplified from $50 \mathrm{ng}$ of normal female DNA and labeled with biotin-dUTP (Roche). The probe mixture was denatured and hybridized to normal metaphase spreads (Vysis, Downers Grove, IL, USA). Unhybridized probes were washed out, and the metaphase spread was incubated with a fluorescein isothiocyanate (FITC)-conjugated anti-DIG antibody (Roche) and rhodamine-conjugated avidin (Roche). Preparations were washed and counterstained with 4,6-diamino-2-phenylinodole (DAPI) in an antifade solution. Red, green, and blue images were acquired, and ratios of fluorescence intensity along chromosomes were quantitated using the ISIS ${ }^{\circ}$ CGH Analysis software (MetaSystems, Heidelberg, Germany). 
Mutation of the IDH genes and methylation of the MGMT promoter were assessed for gliomas. IDH mutations were assessed in three steps. First, FFPE sections were examined for IDH1 R132H by immunohistochemistry with an anti-mutant IDH1 antibody (Dianova, Hamburg, Germany) [3]. For negative cases, exon 4 of the IDH1 gene was amplified and sequenced with previously described primers [25]. For the cases lacking the IDH1 mutation, exon 4 of the $I D H 2$ gene was amplified and sequenced with previously described primers [25].

Methylation status of the MGMT promoter was determined by performing MSP using the EZ DNA Methylation-Direct ${ }^{\text {TM }}$ Kit (Zymo Research Corp., Orange, CA, USA), as described previously [7].

\section{Results}

Histopathological diagnoses of the 12 tumors included five gliomas (two cases of diffuse astrocytoma, IDH-wild type, one case of anaplastic astrocytoma, IDH mutant, one case of glioblastoma, IDH-mutant, and one case of glioblastoma, IDH-wild type), three meningothelial meningiomas (located in the cavernous sinus, olfactory groove, and sphenoid ridge), two chordomas, and two schwannomas according to the WHO criteria in 2016 [13]. The patients' ages ranged from 27 to 41 years. CGH profiles were obtained for all cases, and immunohistochemistry for ER, PgR, AR, EGFR, HER-2, VEGFR-1/2, and cMyc were successfully performed in all cases, while the MIB-1 proliferative index was obtained in 11 of 12 cases.

Molecular-genetic results

Among the five gliomas, AR was expressed in three, ER in one, EGFR in four, VEGFR-1/2 in 4/4, and c-Myc in four cases, while expression of PgR and HER-2 was not detected. Among the three meningiomas, ER was expressed in two, PgR in three, EGFR in one, HER-2 in two, and VEGFR-1/2 in 2/2 cases. Among the two chordomas, EGFR was expressed in one, VEGFR-1/2 in 2/1, and c-Myc in one case. Among the two vestibular schwannomas, VEGFR-1 was expressed in two cases (Table 1). Therefore, there appeared to be some tendency in receptor expression specific to tumor types; female hormone receptors and HER-2 were frequently and exclusively expressed in meningiomas, AR and c-Myc were also frequently and exclusively expressed in gliomas, and EGFR was predominantly expressed in gliomas, whereas expression of VEGFRs was frequently observed in all tumor types.

There were no specific CNAs shared between pregnancy-associated brain tumors (Table 1). MIB-1 indices of pregnancy-associated brain tumors were largely similar to those of each tumor type; for example, the average MIB-1 index of non-pregnant meningothelial meningioma was $1.8 \%$ in our series. However, some high-grade gliomas showed rapid, aggressive development with large tumor size and high MIB-1 indices (see illustrative case below).

Regarding the timing of tumor manifestation in relation to the stage of pregnancy, four of the five gliomas and both schwannomas manifested in the second trimester (Table 2). Interestingly, the expression of VEGFR-1 was shared in all seven tumors that manifested in the second trimester. 
Conversely, all three meningiomas manifested in the later stages of pregnancy, in the third trimester or postpartum period. Sex hormone receptors were shown to be expressed in four of the five tumors that manifested in the third trimester or postpartum period.

Illustrative case

\section{Case 3}

A 30-year-old woman presented with epileptic seizures and magnetic resonance imaging (MRI) revealed a high-intensity lesion on T2-weighted images involving her left frontotemporal operculum and insula (Fig. 1A). She underwent biopsy and pathological diagnosis was diffuse astrocytoma and an IDH-mutant [13]. Her tumor gradually progressed, and nine months after the initial biopsy, she underwent the second surgery of partial resection (70\%) with an awake procedure (Fig. 1B). The pathological diagnosis was diffuse astrocytoma, and IDH-mutant [13]. Chromosomal number analysis by CGH revealed -4 pter-15.3, -6q16, +8q21.3-ter (including c-Myc locus), -19q13.13-ter and -X. MGMT promoter was unmethylated, and MIB-1 index was 3.5\%. She was observed in a watchful wait policy afterwards. Thirteen months after the second surgery, positron emission tomography (PET) revealed methionine uptake in her left temporal lobe (Fig. 1C, D, E). However, she was found to be pregnant (six weeks of gestation) immediately after the PET scan, and strongly wished to continue pregnancy. She was checked for symptoms every two to three months, without radiological assessment due to lack of complaints. However, she presented with a strong headache, nausea and right hemiparesis at 23 weeks and 3 days of gestation, and MRI revealed a large contrast-enhancing lesion with extensive perifocal edema (Fig. 1F, G). She underwent an emergent tumor resection with maintenance of pregnancy, and the pathological diagnosis was anaplastic astrocytoma, IDH-mutant [13]. The tumor had numerous CNAs including gain of MYClocus on 8q24 (Fig. 2B, 2C). The MGMT promoter was unmethylated and the MIB-1 index was $84.5 \%$. Immunohistochemistry of the tumor showed expression of AR, EGFR, c-Myc, VEGFR-1, and VEGFR-2 expression (Fig. 3). At 34 weeks of gestation, follow-up MRI revealed enlargement of the tumor $($ Fig. $1 \mathrm{H}, \mathrm{I})$. Therefore, she was scheduled to undergo cesarean section at 35 weeks of gestation and delivered a healthy baby. Three days after delivery, she underwent her fourth surgery of gross total resection of the contrast-enhanced lesion with an awake procedure. The pathological diagnosis was anaplastic astrocytoma, an IDH-mutant [13]. Subsequently, she received radiation therapy (60Gy/30Fr) with concurrent and adjuvant temozolomide. Five months after the 4th surgery, MRI revealed a new, nodular contrast-enhancement in her insula (Fig. $1 \mathrm{~J}, \mathrm{~K}$ ). She underwent her 5 th surgery, and the contrast-enhanced lesion was only partially resected due to a decline in motor evoked potential. The pathological diagnosis was diffuse astrocytoma and an IDH-mutant [13]. The CGH profile of the tumor was reminiscent of those of the 2 nd and $3 r d$ resections; however, the MYC locus appeared only gained, not amplified (Fig. 2D). Afterwards, she received peptide vaccine therapy including those derived from VEGFR-1 and 2, and there was no evidence of tumor recurrence five years and six months after the 5th surgery (Fig. 1L). Six years and seven months later at the time of manuscript writing, the child was healthy without any congenital malformation or growth retardation. 


\section{Discussion}

The mechanism by which pregnancy affects brain tumors has not yet been clearly elucidated. One proposed mechanism is that the increase in systemic and cerebral blood volume that normally occurs during pregnancy may increase peritumoral edema and absolute tumor blood volume [2]. Indeed, in some cases of low-grade gliomas showing progression during pregnancy, the radiological velocity of diametric expansion (VDE) returned to pre-pregnancy values after delivery [16]. However, many cases have been documented that transformation from low-grade gliomas to high-grade gliomas (WHO grade 3 and 4) occurred in women during pregnancy [5]. Therefore, progression of brain tumors in this setting may not only be associated with an increase in systemic and cerebral blood volume, but also with other unidentified mechanisms such as the activation of specific receptors by maternal hormones and various growth factors.

It is known that estrogen and progesterone increase during pregnancy, especially in the second and third trimesters, followed by androgen. Progesterone stimulates embryo implantation, endometrial hypertrophy, and development of mammary glands. Estrogen stimulates the development of the uterus, embryo development, and hypertrophy of prolactin producing lactotrophs. EGF increases during early pregnancy and stimulates placental growth. EGFR is a prototypical member of the ErbB/EGFR family, which is involved in multiple cellular processes, including migration, adhesion, differentiation, and apoptosis, and critically regulates the growth and survival of the endometrium in response to embryo implantation during early pregnancy [11]. HER-2 is known as c-erbB-2, and PgR, ER, and HER-2 are known as significant prognostic factors in pregnancy-associated breast cancer patients. Placental expression of VEGF-A is intense and expression of placental growth factor (PIGF) is moderate during early pregnancy[VEGF-A binds to two related tyrosine kinase receptors expressed on vascular endothelial cells, VEGFR-1 and VEGFR-2. PIGF is a member of the VEGF family and specifically binds to VEGFR-1. These promote angiogenesis and stimulate stromal cells and placental vasculature development. From 25 weeks of gestation, angiogenesis switches from branching to non-branching and is accompanied by a decline in VEGF-A and increase in PIGF [1].

Regarding the mechanism of individual brain tumor types, growth of meningioma during pregnancy is known to be related to sex hormones. The positive cell ratios for ER and PgR in meningiomas are high and $\mathrm{PgR}$ is more widely detected than ER. It is reported that meningothelial meningiomas most frequently express PgR, while fibrous meningiomas most frequently express ER [19]. The expression of HER-2, ER, and $\mathrm{PgR}$ is known to be involved in the development of pregnancy-associated breast cancer [18]. Likewise, HER-2 expression was detected only in meningiomas that expressed PgR and/or ER in the present study. Among the three meningiomas in the present study, one developed in the 3rd trimester, and two immediately after giving birth (Table 2). Importantly, all three tumors were meningothelial meningiomas, most of which expressed PgR. As estrogen and progesterone increase especially in the 2nd and 3rd trimesters, the development of meningioma during the late stage of pregnancy is likely attributable to signal transduction via these hormone receptors. In addition, overexpression of HER-2 can stimulate meningioma cell proliferation and invasion via its two main signaling pathways: the PI3K/AKT 
and RAS/MAPK pathways [24]. Phospholylated Erk1/2 MAPK, a signaling molecule in the EGFR/HER-2 pathway, is significantly reduced by estrogen deprivation but is increased by estrogen in breast cancers, confirming the crosstalk between ER and HER-2 [15] .

As for gliomas, AR was found to be positive in three of five gliomas, c-myc was positive in two, equivocal in two, and EGFR and VEGFR-1/2 were expressed in four of the five gliomas that progressed during pregnancy. AR was shown to be frequently expressed at high levels in high-grade gliomas [17]. EGFR is a pivotal driver in both the initiation of primary glioblastoma and progression of lower-grade glioma to glioblastoma [9]. VEGF-A and PIGF play a significant role in the maintenance of pregnancy, and is also a well-known, key angiogenic factor for the progression of gliomas $[5,23]$. Regarding the timing of tumor manifestation, four of the five gliomas presented in the 2nd trimester, in line with a previous study reporting that tumor growth of gliomas occurs in the early stage of pregnancy. Indeed, VEGF-A and EGF increase particularly in the early stage of gestation [11] and the increase of PIGF begins in the 2nd trimester [1], Moreover, the predominant occurrence of gliomas in the 2nd trimester is reasonably attributable to the increase of those growth factors.

It is noteworthy that MYC amplification was clearly demonstrated on $\mathrm{CGH}$ in the high-grade glioma resected during pregnancy in case 3, but not in either the precedent low-grade tumor or the relapsed tumor thereafter (Fig. 2). MYC is a proto-oncogene located on chromosome $8 q 24$, which is involved in cell cycle regulation, proliferation, and development of cancer. Overexpression of its protein product, c-Myc, has been observed in many cancer types and leads to the upregulation of many genes related to cell proliferation. Importantly, c-Myc expression is also known to be upregulated in the early gestation placenta, but sparse or absent at term [6]. Therefore, MYC amplification noted only in the tumor resected during pregnancy may be associated with the molecular environment in early gestation. Overexpression of c-Myc could be among the driver mechanisms of progression of gliomas during the early stage of pregnancy, especially those with $8 q$ gain.

As for schwannoma, a previous study reported 31 cases of vestibular schwannoma that were diagnosed or became symptomatic during pregnancy. In that series, the most frequent presenting symptom was hearing loss, and all cases except two were diagnosed in the second or third trimester of pregnancy [22]. In line with the previous study, both cases in this study were presented in the late 2nd trimester. Importantly, in the present study, VEGFR-1 was the only growth factor/hormone receptor detected in the schwannomas in the two cases. Therefore, the upregulation of VEGF-A, particularly in the early gestational stage and increase of PIGF beginning in the 2nd trimester is likely associated with the gradual increase of the benign tumor that might manifest in the $2 \mathrm{nd}$ or $3 \mathrm{rd}$ trimester.

To our knowledge, there have been no reports of pregnancy-associated chordoma in the literature. In the two chordomas presented here, one with 2 + expression of VEGFR-2 presented in the early 2 nd trimester, and the other one was positive for EGFR and c-Myc and presented immediately after giving birth.

As mentioned above, VEGF-A and EGF increase throughout the gestation period with the peak in the early stage and increase of PIGF begins in the 2 nd trimester $[1,11]$. Therefore, high-grade tumors with receptors 
for these growth factors could manifest with acceleration of proliferation and/or malignant transformation in the relatively early stage, and low-grade or benign tumors might present following gradual volume increase in the later stage of pregnancy. In contrast, because serum levels of sex hormones increase during pregnancy, especially in the second and third trimesters, tumors with hormone receptors such as meningiomas tend to be present during the late pregnancy periods. Indeed, in the present study, ER, PgR, and HER-2 were exclusively expressed in tumors (meningiomas) that were present in the 3rd trimester or postpartum period. Conversely, other receptors/molecules, especially VEGFR-1/2 and EGFR, were found to be expressed regardless of the timing of tumor manifestation.

In conclusion, growth of brain tumors can be accelerated during pregnancy due to the upregulation of various growth factors as well as sex hormones. Tumor type and their receptors appear to be associated with the timing of presentation.

\section{Abbreviations}

AR: androgen receptor; ER: estrogen receptor; IDH: isocitrate dehydrogenase; MGMT: $0^{6}$-methylguanine DNA methyltransferase; PgR: progesterone receptor; EGFR: epidermal growth factor receptor; VEGFR: Vascular endothelial growth factor receptor; HER-2: Human epidermal growth factor receptor 2

\section{Declarations}

\section{Acknowledgements}

The authors thank Ms.Naoko Tsuzaki for her technical assistance.

\section{Funding}

This study was funded by Grant-in-Aid for Scientific Research (KAKENHI) by The Ministry of Education, Culture, Sports, Science and Technology and The Japan Society for the Promotion of Science (Grant Number 16K20025).

\section{Conflict of Interests}

The authors report no conflict of interests concerning the materials or methods used in this study or the findings specified in this article.

\section{Availability of data and material}

All data generated or analysed during this study are included in this published article.

\section{Author's contributions}

All authors contributed to the study conception and design. Conceptualization: Kazunari Yoshida; Methodology: Hikaru Sasaki; Resources: Maya Kono, Katsuhiro Mizutani, Isako Saga, Takenori Akiyama, 
Masahiro Toda, Kazunari Yoshida, Michihiro Kohno, Hikaru Sasaki; Formal analysis: Saeko Ichimura, Kentaro Ohara; Data curation, investigation, visualization, funding acquisition, writing - original draft preparation: Saeko Ichimura; Writing - review and editing: Hikaru Sasaki, Ryuichi Kanai, Yohei Kitamura; Supervision: Hikaru Sasaki. All authors commented on previous versions of the manuscript and approved the final manuscript.

\section{Ethical approval}

All procedures performed in the present study were in accordance with the ethical standards of the institutional and /or national research committee and with the 1964 Helsinki declaration and its later amendments or comparable ethical standards.

\section{Consent to participate}

Informed consent was obtained from all individual participants including in the study.

\section{Consent to publication}

Patients signed informed consent regarding publishing their data and photographs.

\section{Informed consent}

Informed consent was obtained from all individual participants included in the study

\section{References}

1. Andraweera PH, Dekker GA, Roberts CT (2012) The vascular endothelial growth factor family in adverse pregnancy outcomes. Human Reproduction Update 18:436-457. doi:10.1093/humupd/dms011

2. Blumenthal DT, Parreño MGH, Batten J, Chamberlain MC (2008) Management of malignant gliomas during pregnancy. Cancer 113:3349-3354. doi:10.1002/cncr.23973

3. Capper D, Zentgraf H, Balss J, Hartmann C, von Deimling A (2009) Monoclonal antibody specific for IDH1 R132H mutation. Acta Neuropathologica 118:599. doi:10.1007/s00401-009-0595-Z

4. Cushing H, Eisenhardt L (1938) Meningiomas. Their Classification, regional behaviour, life history, and surgical end results. Bulletin of the Medical Library Association 27:185-185

5. Daras M, Cone C, Peters KB (2014) Tumor progression and transformation of low-grade glial tumors associated with pregnancy. Journal of Neuro-Oncology 116:113-117. doi:10.1007/s11060-013-12619

6. Diebold J, Arnholdt H, Lai MD, Löhrs U (1992) C-myc expression in early human placenta -a critical evaluation of its localization. Virchows Archiv B 61:65. doi:10.1007/BF02890406

7. Ezaki T, Sasaki H, Hirose Y, Miwa T, Yoshida K, Kawase T (2011) Molecular characteristics of pediatric non-ependymal, non-pilocytic gliomas associated with resistance to temozolomide. 
Molecular Medicine Reports 4:1101-1105

8. Hirose Y, Aldape K, Takahashi M, Berger MS, Feuerstein BG (2001) Tissue mocrodissection and degenerate oligonucleotide primed-polymerase chain reaction (DOP-PCR) is an effective method to analyze genetic aberrations in invasive tumors. The Journal of Molecular Diagnostics 3:62-67. doi:http://dx.doi.org/10.1016/S1525-1578(10)60653-8

9. Huang PH, Xu AM, White FM (2009) Oncogenic EGFR signaling networks in glioma. Science Signaling 2:re6-re6. doi:10.1126/scisignal.287re6

10. Kitamura Y, Sasaki H, Kimura T, Miwa T, Takahashi S, Kawase T, Yoshida K (2013) Molecular and clinical risk factors for recurrence of skull base chordomas: Gain on chromosome 2p, expression of brachyury, and lack of irradiation negatively correlate with patient prognosis. Journal of Neuropathology \& Experimental Neurology 72:816-823

11. Large MJ, Wetendorf M, Lanz RB, Hartig SM, Creighton CJ, Mancini MA, Kovanci E, Lee K-F, Threadgill DW, Lydon JP, Jeong JW, DeMayo FJ (2014) The epidermal growth factor receptor critically regulates endometrial function during early pregnancy. PLOS Genetics 10:e1004451. doi:10.1371/journal.pgen.1004451

12. Louis DN, Ohgaki H, Wiestler OD, Cavanee WK (2007) WHO classification of tumours of the central nervous system.

13. Louis DN, Ohgaki H, Wiestler OD, Cavanee WK, Ellison DW, Figarella-Branger D, Perry A, Reifenberger Guido, von Deimling A (2016) WHO classification of tumours of the central nervous system. IARC

14. Miwa T, Hirose Y, Sasaki H, Ezaki T, Yoshida K, Kawase T (2011) Single-copy gain of chromosome 1q is a negative prognostic marker in pediatric nonependymal, nonpilocytic gliomas. Neurosurgery 68:206-212. doi:10.1227/NEU.0b013e3181fd2c2e

15. Osborne CK, Shou J, Massarweh S, Schiff R (2005) Crosstalk between estrogen receptor and growth factor receptor pathways as a cause for endocrine therapy resistance in breast cancer. Clinical Cancer Research 11:865s

16. Pallud J, Mandonnet E, Deroulers C, Fontaine D, Badoual M, Capelle L, Guillet-May F, Page P, Peruzzi P, Jouanneau E, Frenay M, Cartalat-Carel S, Duffau H, Taillandier L (2010) Pregnancy increases the growth rates of world health organization grade II gliomas. Annals of Neurology 67:398-404. doi:10.1002/ana.21888

17. Paoletti P BG, Zibera C (1990) Characteristics and biological role of steroid hormone receptors in neuroepithelial tumors. Journal of Neurosurgery 73:736-742. doi:10.3171/jns.1990.73.5.0736

18. Reed W, Hannisdal E, Skovlund E, Thoresen S, Lilleng P, Nesland JM (2003) Pregnancy and breast cancer: a population-based study. Virchows Archiv 443:44-50. doi:10.1007/s00428-003-0817-z

19. Roelvink NA, Kamphorst W, van Alphen HM, Rao B (1987) Pregnancy-related primary brain and spinal tumors. Archives of Neurology 44:209-215. doi:10.1001/archneur.1987.00520140069020

20. Saitoh Y, Oku Y, Izumoto S, Go J (1989) Rapid growth of a meningioma during pregnancy: Relationship with estrogen and progesterone receptors; Case report. Neurologia medico-chirurgica 29:440-443. doi:10.2176/nmc.29.440 
21. Sasaki H, Zlatescu MC, Betensky RA, Ino Y, Cairncross JG, Louis DN (2001) PTEN is a target of chromosome $10 \mathrm{q}$ loss in anaplastic oligodendrogliomas and PTEN alterations are associated with poor prognosis. The American Journal of Pathology 159:359-367.

doi:http://dx.doi.org/10.1016/S0002-9440(10)61702-6

22. Shah KJ, Chamoun RB (2014) Large vestibular schwannomas presenting during pregnancy: Management strategies. Journal of Neurological Surgery Part B, Skull Base 75:214-220. doi:10.1055/s-0034-1370784

23. Tamura R, Tanaka T, Miyake K, Yoshida K, Sasaki H (2017) Bevacizumab for malignant gliomas: current indications, mechanisms of action and resistance, and markers of response. Brain Tumor Pathology 34:62-77. doi:10.1007/s10014-017-0284-x

24. Wang Z, Wang W, Xu S, Wang S, Tu Y, Xiong Y, Mei J, Wang C (2016) The role of MAPK signaling pathway in the Her-2-positive meningiomas. Oncol Rep 36:685-695. doi:10.3892/or.2016.4849

25. Yan H, Parsons DW, Jin G, McLendon R, Rasheed BA, Yuan W, Kos I, Batinic-Haberle I, Jones S, Riggins GJ, Friedman H, Friedman A, Reardon D, Herndon J, Kinzler KW, Velculescu VE, Vogelstein B, Bigner DD (2009) IDH1 and IDH2 mutations in gliomas. New England Journal of Medicine 360:765773. doi:10.1056/NEJMoa0808710

\section{Tables}

Due to technical limitations, table 1 and 2 is only available as a download in the Supplemental Files section.

\section{Figures}




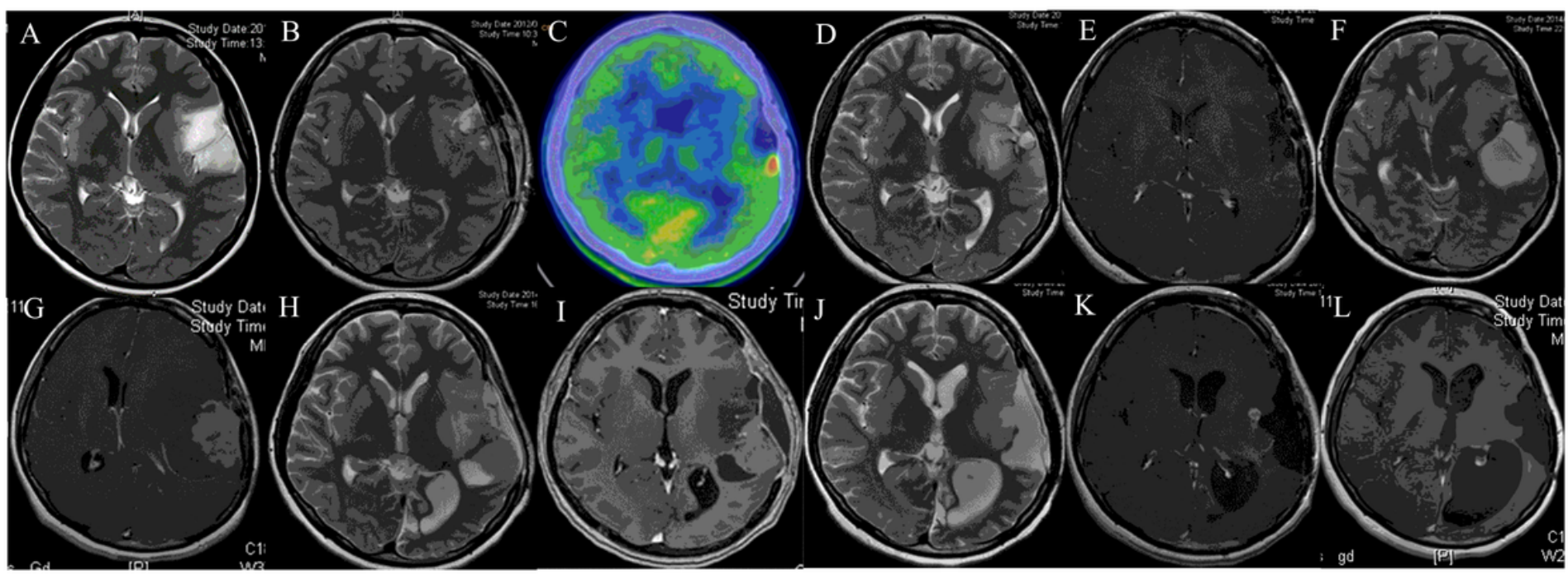

\section{Figure 1}

Serial radiological images of case 3. A, B, D, F, H, J: T2-weighted MRI. C: C11-methionine PET. E, G, I, K: T1weighted MRI with gadolinium administration. A: Before the 2nd surgery. B: After the 2nd surgery (partial resection with the awake procedure). C-E: At the notification of pregnancy ( 3 weeks and 2 days of gestation, 2 years and 1 month after the 2 nd surgery. Although there was no definite evidence of tumor recurrence on T2 or contrast-enhanced T1 images, C11-methionine PET suggested tumor recurrence in the left temporal lobe. F, G: At the worsening of the patient's symptoms ( 23 weeks 3 days of gestation). MRI revealed a large contrast-enhancing lesion in her left temporal lobe. Subsequently, the patient underwent a subtotal tumor resection with maintenance of pregnancy. $\mathrm{H}, \mathrm{l}$ : At 34 weeks of gestation, follow-up MRI revealed enlargement of the tumor. Gross total removal was performed following cesarean section (4th surgery). J, K: Five months after the 4th surgery, MRI revealed a new, nodular contrastenhancement in the left insula, and partial resection of the tumor was performed (5th surgery). L: The patient had been treated with peptide vaccine therapy following the 5th surgery, and there was no evidence of tumor recurrence 5 years and 2 months after the 5th surgery ( 9 years and 2 months after the initial biopsy). 


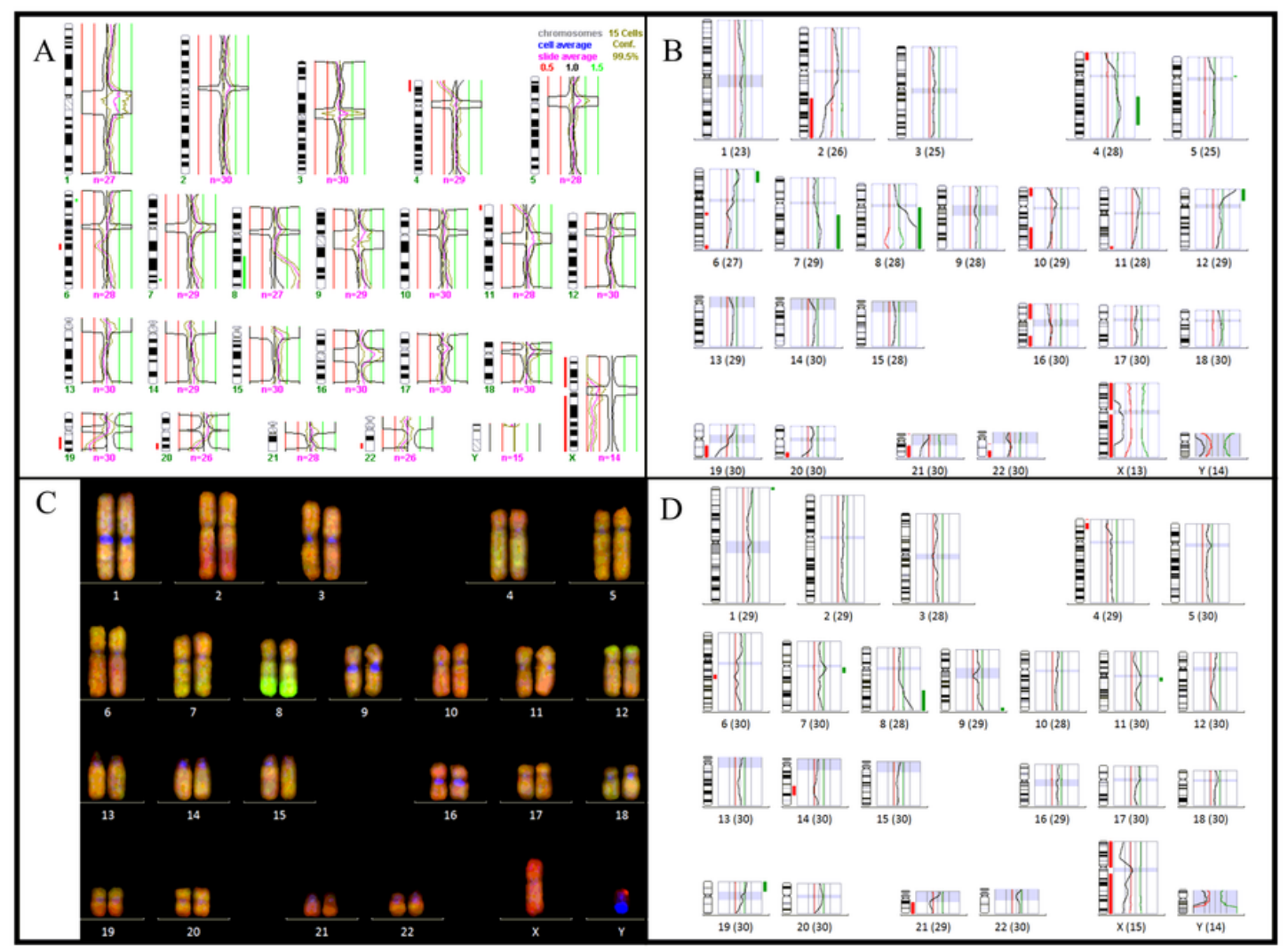

Figure 2

Chromosomal copy number aberrations (CNAs) of case 3 by metaphase comparative genomic hybridization. A, CNA profile of the 2 nd resection. Red lines to the left of each idiogram represent regions of reduced relative DNA copy number, and green lines to the right represent regions of increased relative DNA copy number. B, CNA profile of the 3rd resection during pregnancy. Note that 8q24 was amplified. C, Color illustration of CNAs of the 3rd resection on lymphocyte spread. Red indicates relative loss of chromosomal region, and green indicates relative gain. D, CNA profile of the 5th resection. Although $8 q 24$ is gained, but not amplified. 


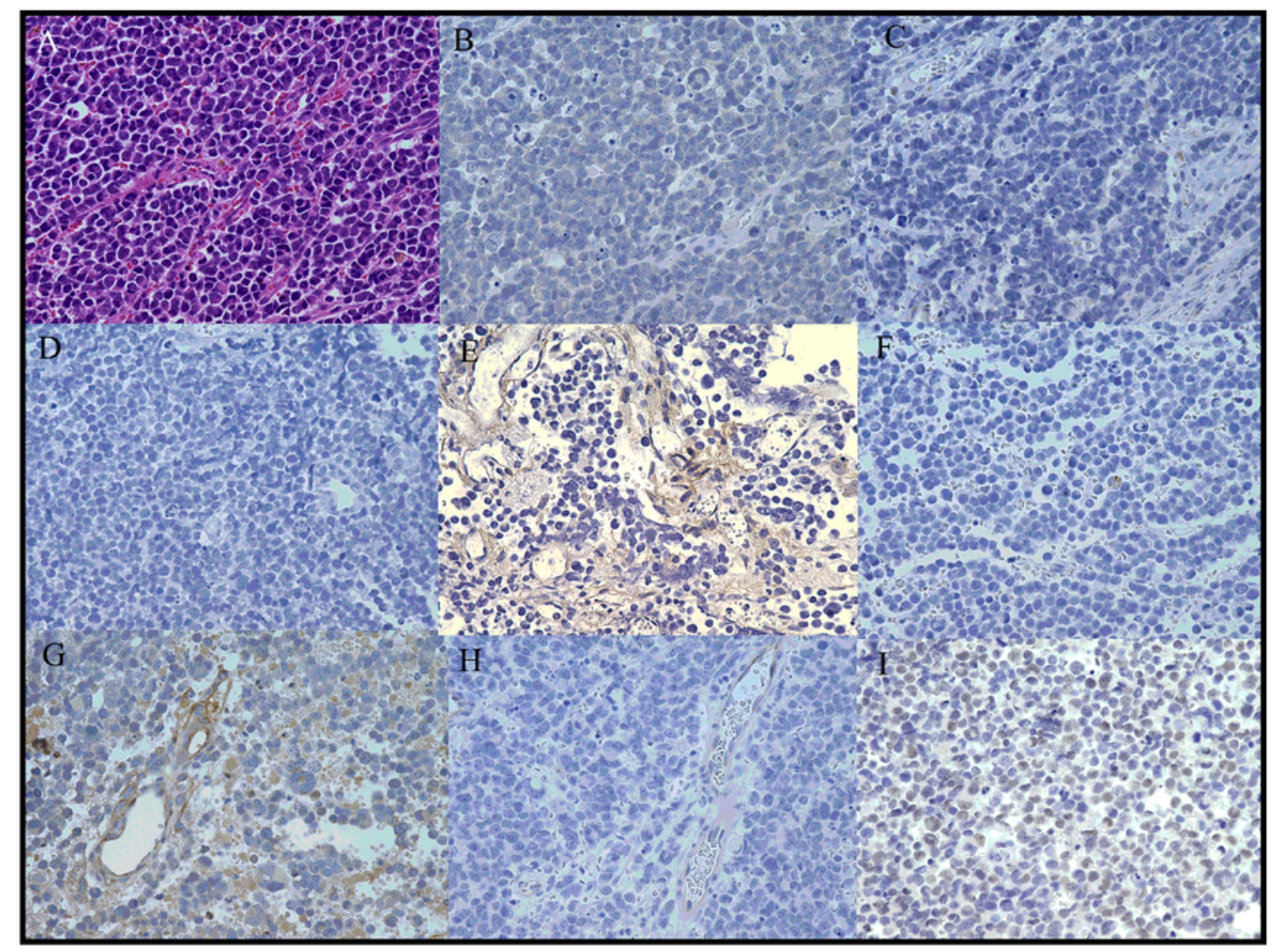

\section{Figure 3}

Photomicrograph of the tumor of the 3rd resection of case 3. (A) Hematoxylin and eosin staining. (B) Staining of AR. Most of the tumor cells were positive for AR. (C) Staining of ER (negative). (D) Staining of PgR (negative). (E) Staining of EGFR. Many tumor cells were positive for EGFR. (F) Staining of HER-2 (negative). (G) Staining of VEGFR-1. VEGFR-1 was strongly positive on many tumor cells as well endothelial cells. (H) Staining of VEGFR-2. VEGFR-2 was weakly positive on endothelial cells. (I) Staining of c-Myc. Most tumor nuclei were positive for c-Myc. Original magnification $\times 400$.

\section{Supplementary Files}

This is a list of supplementary files associated with this preprint. Click to download.

- PregnancyTable1.pdf

- PregnancyTable2.pdf 